Tema: Metalurgia Secundária

\title{
INFLUÊNCIA DA COMPOSIÇÃO DA ESCÓRIA DE PANELA NA DISSOLUÇÃO DO COATING $\left(\mathrm{C}_{2} \mathrm{~S}\right)$ *
}

\section{Resumo}

Ricardo Thomé da Cruz ${ }^{1}$ Gustavo Frassini Pelisser ${ }^{2}$

Wagner Viana Bielefeldt ${ }^{3}$ Saulo Roca Bragança ${ }^{4}$

$\mathrm{Na}$ produção de aços é essencial garantir maior durabilidade do material refratário em panelas siderúrgicas. A formação de um revestimento protetor ("coating") de silicato dicálcico $\left(\mathrm{C}_{2} \mathrm{~S}\right)$ em tijolos dolomíticos em contato com escórias básicas é fundamental ao aumento da vida útil dos mesmos. As temperaturas solidus e liquidus do $\mathrm{C}_{2} \mathrm{~S}$ alfa puro são de aproximadamente $1.464^{\circ} \mathrm{C}$ e $2.154^{\circ} \mathrm{C}$. Assim sendo, é esperada uma alta fração de sólido nas temperaturas de refino do aço. No entanto, a presença de óxidos como $\mathrm{Al}_{2} \mathrm{O}_{3}, \mathrm{FeO}$ e a fluorita $\left(\mathrm{CaF}_{2}\right)$ na escória podem acelerar a dissolução do coating pela formação de fases de menor temperatura de fusão. A fim de melhor compreender esse processo, foram preparadas amostras de diferentes composições de fluorita $+\mathrm{C}_{2} \mathrm{~S}$ na temperatura de $1.600^{\circ} \mathrm{C}$. Os resultados foram analisados com o auxilio do software FactSage (simulação termodinâmica). Especificamente, foi estudada a influência da formação de fases e da temperatura de formação das mesmas, as quais levam a fusão do coating. Os dados de simulação foram validados através de ensaios em forno de laboratório.

Palavras-chaves: Refratários dolomíticos; Coating; Corrosão; FactSage.

\section{SLAG LADLE COMPOSITION INFLUENCE IN THE DISSOLUTION OF COATING $\left(\mathrm{C}_{2} \mathrm{~S}\right)$}

\section{Abstract}

In steel making is vital to ensure durability of the refractory material in ladle. The formation of a protective coating of dicalcium silicate $\left(\mathrm{C}_{2} \mathrm{~S}\right)$ in dolomite bricks in contact with basic slag is critical to increasing their useful life. The solidus and liquidus temperatures of the pure alpha $\mathrm{C}_{2} \mathrm{~S}$ are approximately $1,464^{\circ} \mathrm{C}$ and $2,154^{\circ} \mathrm{C}$. Thus, it is expected a high solid fraction at temperatures of steel refining. However, the presence of oxides such as $\mathrm{Al}_{2} \mathrm{O}_{3}, \mathrm{FeO}$ and fluorite $\left(\mathrm{CaF}_{2}\right)$ in the slag can accelerate the dissolution of the coating by the formation of phases of lower melting temperature. In order to better understand this process, samples were prepared with different compositions of fluorite $+\mathrm{C}_{2} \mathrm{~S}$ at a temperature of $1,600^{\circ} \mathrm{C}$. The results were analyzed with the help of software FactSage (thermodynamic simulation). Specifically, were studied the influence of the phases formation and the temperature of the phases, which leads to fusion of the coating. The simulation data were validated through testing in a laboratory furnace.

Keywords: Dolomitic refractories; Coating; Corrosion; Factsage.

Doutorando, PPGE3M, UFRGS, Poá, RS, Brasil.

Mestrando, PPGE3M, UFRGS, Poá, RS, Brasil.

Engenheiro Metalúrgico, Doutor, Professor, PPGE3M, UFRGS, Poá, RS, Brasil.

Engenheiro Químico, Doutor, Professor, PPGE3M, UFRGS, Poá, RS, Brasil.

\footnotetext{
* Contribuição técnica ao 45 Seminário de Aciaria - Internacional, 25 a 28 de maio de 2014
} Porto Alegre, RS, Brasil. 


\section{INTRODUÇÃO}

O avanço tecnológico dos materiais refratários aplicados em siderurgia está diretamente relacionado com uma significativa redução de custos e aumento da segurança operacional nos processos siderúrgicos [1]. Em um mercado mundial competitivo e integrado é necessário um profundo conhecimento de refratários, para sua correta seleção e aplicação, como forma de reduzir os custos associados, garantido também maior vida útil dos mesmos em operação.

Em aciarias semi-integradas, com aços acalmados ao silício, os refratários dolomíticos têm sido amplamente utilizados. Eles apresentam excelente compatibilidade com as escórias básicas, são resistentes ao choque térmico e apresentam elevada refratariedade [2].

$\mathrm{A}$ boa resistência às escórias dos refratários doloma- $\mathrm{C}$ deve-se à presença de $\mathrm{CaO}$ livre, não encontrado em outros materiais refratários de menor basicidade. Quando em contato com escórias básicas, não completamente saturadas em $\mathrm{CaO}$, a escória começa a dissolver o $\mathrm{CaO}$ do tijolo, formando um líquido de alta basicidade $\mathrm{e}$ viscosidade, até a formação de uma camada densa de $\mathrm{C}_{2} S$ [3]. A formação da fase $\mathrm{C}_{2} \mathrm{~S}\left(\mathrm{Tf}=2130^{\circ} \mathrm{C}\right)$ [4] na interface refratário escória retarda a dissolução da fase $\mathrm{CaO}$ pela ação da escória líquida, uma vez que essa fase forma uma fina camada (denominada coating) que protege o refratário contra a penetração da escória.

Lee e Zhang [5] revisaram os tipos de ataques de escórias líquidas em refratários. Segundo os autores, o ataque pode ser direto ou indireto. Se o produto da reação é solúvel ou dissocia diretamente na escória líquida, o ataque é direto (corrosão ativa) e pode continuar destruindo o refratário. No entanto, se o produto da reação não é completamente solúvel na escória líquida, pode ocorrer à formação de uma barreira que após a sua constituição impede ataques posteriores. Neste caso, o ataque é dito indireto (corrosão passiva) e a taxa de corrosão passa a ser controlada pela velocidade da reação química, difusão através da camada de $\mathrm{C}_{2} \mathrm{~S}$ ou difusão dos componentes através da escória.

A grande vantagem do coating de superfície é que a camada é formada através de uma reação química termodinamicamente favorável, com forte aderência no tijolo. Após a formação do coating, a presença de óxidos como $\mathrm{Al}_{2} \mathrm{O}_{3}, \mathrm{FeO}$ e a fluorita podem dissolver o coating, formando fases de menor temperatura de fusão, como aluminatos e silicatos de $\mathrm{Ca}$ (com ou sem outros óxidos ou minerais como a failita). A fluorita é uma matéria-prima largamente utilizada na produção do aço, devido a sua grande capacidade de diminuir a viscosidade, aumentar a fração de fase líquida, e, consequentemente, aumentar a cinética de reação para as escórias no refino secundário, diminuindo o teor de enxofre no aço [6].

Helbert [7] estudou o efeito do teor de fluorita e da concentração de $\mathrm{CaO}$ na taxa de desgaste de tijolos de magnesianos em $1650^{\circ} \mathrm{C}$. Os resultados deste estudo expõem a grande importância da saturação da escória em CaO. Além disso, indicam o risco do desgaste excessivo de refratários em decorrência do uso sem controle de fluorita, a qual diminui a temperatura de fusão e a viscosidade das escórias, tornando-as mais agressivas. Portanto, a formação/manutenção do coating depende da saturação da escória em $\mathrm{CaO}$, do uso controlado de fluorita e da neutralização dos sesquióxidos.

A fim de melhor compreender esse processo, foram realizados experimentos em laboratório com amostras de diferentes composições de fluorita $+\mathrm{C}_{2} \mathrm{~S}$ a $1600^{\circ} \mathrm{C}$. Um estudo termodinâmico de previsão de fases foi realizado com o auxilio do software FactSage (simulação termodinâmica). Especificamente, foi estudada a influência da

\footnotetext{
* Contribuição técnica ao 45 Seminário de Aciaria - Internacional, 25 a 28 de maio de 2014, Porto Alegre, RS, Brasil.
} 
formação de fases e da temperatura de formação das mesmas, as quais levam a fusão do coating. Os dados de simulação foram validados com os ensaios de laboratório e trabalhos da literatura técnica.

\section{MATERIAIS E MÉTODOS}

A dissolução da camada protetora de $\mathrm{C}_{2} \mathrm{~S}$ em revestimentos dolomíticos foi analisada em função da concentração de fluorita. Diferentes composições do $\mathrm{C}_{2} \mathrm{~S}$ com teores crescentes de fluorita foram estudadas, vide Tabela 1. Este estudo teve como base uma amostra de coating retirada de uma panela siderúrgica em condições normais de processo e uma amostra de fluorita procedente de um lote industrial.

Tabela 1. Relações em \% em massa de $\mathrm{C}_{2} \mathrm{~S}$ e $\mathrm{CaF}_{2}$ avaliadas neste trabalho

\begin{tabular}{ccc}
\hline Amostra & Sistema & $\begin{array}{c}\text { composição } \\
(\%-M)\end{array}$ \\
\hline A & C2S - CaF2 & $100-0$ \\
B & C2S - CaF2 & $95-5$ \\
C & C2S - CaF2 & $90-10$ \\
D & C2S - CaF2 & $80-20$ \\
\hline
\end{tabular}

A avaliação do comportamento térmico das composições apresentadas na Tabela 1 foi realizada através do uso do software de simulação termodinâmica (programa FactSage) [8]. Os cálculos realizados pelo programa são baseados na minimização da energia livre do sistema e fornecem informações sobre as fases formadas, suas proporções e composições, as atividades individuais de cada componente químico e as propriedades termodinâmicas para várias composições, pressões e temperaturas [9]. É importante ressaltar que todos os cálculos realizados na simulação termodinâmica basearam-se na composição química da amostra de $\mathrm{C}_{2} \mathrm{~S}$ retirada da panela de aço (Tabela 2).

Uma descrição geral do programa e bancos de dados pode ser visto em Bale et. al. [9], bem como recentes modificações [10]. Os seguintes bancos de dados foram utilizados, descritos na documentação do programa [11]: O banco de dados de soluções FToxid (FToxid53Soln.sda) contém soluções de óxidos avaliadas pelo grupo que desenvolve o programa. $\mathrm{O}$ banco de dados FToxid compound database (FToxid53Base.cdb) contém sólidos estequiométricos e óxidos líquidos. O conjunto de bancos de dados FToxid incorpora dados para óxidos puros e soluções de 20 elementos (e para soluções diluídas de $\mathrm{S}, \mathrm{SO}_{4}, \mathrm{PO}_{4}, \mathrm{H}_{2} \mathrm{O} / \mathrm{OH}, \mathrm{CO}_{3}, \mathrm{~F}, \mathrm{Cl}, \mathrm{I}, \mathrm{C}, \mathrm{N}$ e $\mathrm{CN}$ em escórias fundidas (líquidas). A escolha de fases foi executada com o auxílio dos resultados de difração de raios $X$. Portanto, as simulações realizadas com 0 auxílio da ferramenta termodinâmica foram validadas através de ensaios práticos realizados em laboratório e estudos bibliográficos. A simulação foi realizada com temperaturas variando de 0 a $1700^{\circ} \mathrm{C}$, em intervalos de $50^{\circ} \mathrm{C}$.

Os ensaios práticos com as amostras da Tabela 1 foram realizados em um forno com atmosfera inerte (argônio) e temperatura de $1600^{\circ} \mathrm{C}$. Devido à alta temperatura do teste, as amostras foram colocadas dentro de cadinhos de grafita, que proporcionam alta condutividade térmica e são inertes na faixa de temperatura e atmosfera utilizadas no teste.

* Contribuição técnica ao 45 Seminário de Aciaria - Internacional, 25 a 28 de maio de 2014, Porto Alegre, RS, Brasil. 
O forno consiste em um tubo de grafita vertical (onde o cadinho é alojado), circundado por uma resistência elétrica tubular, também de grafita. Esse conjunto é revestido por uma camada de isolante térmico (composto por fibras de alumina e de carbono), acondicionado em um cubo de aço inoxidável, o qual forma a carcaça do forno. Tanto o tubo quanto a câmara recebem injeção de argônio de alta pureza. $\mathrm{O}$ forno apresenta como características operacionais: temperatura máxima de operação: $2100^{\circ} \mathrm{C}$; temperatura contínua de operação: $1950^{\circ} \mathrm{C}$; potência aparente: 18 kVA; potência ativa: $16 \mathrm{~kW}$; tensão de operação (no primário) 3x380 V / 50-60 $\mathrm{Hz}$.

Após o teste as amostras foram submetidas à análise de difração de raios $X$ (Philips X'Pert). Assim, foi possível avaliar a evolução das fases formadas em função do teor de fundente e também comparar estes resultados com as simulações termodinâmicas. A análise química da amostra de coating (Tabela 2) foi realizada por fluorescência de raios-X (espectrômetro Shimadzu XRF-1800).

\section{RESULTADOS}

A análise química da amostra do coating retirada da panela em condições normais de processo (Tabela 2), revela que há presença de algumas impurezas $\left(\mathrm{MgO}, \mathrm{SO}_{3}\right.$, $\mathrm{Al}_{2} \mathrm{O}_{3}, \mathrm{Fe}_{2} \mathrm{O}_{3}$ ), além dos óxidos predominantes de cálcio e silício. Segundo Gomes [12], o $\mathrm{C}_{2} \mathrm{~S}$ pode apresentar de 4 a $6 \%$ em massa de outros elementos em solução sólida. Em processos envolvendo alto teor de $\mathrm{SO}_{3} \mathrm{O}$ cristal de $\mathrm{C}_{2} \mathrm{~S}$ tende a concentrar parte deste óxido em sua estrutura. Isto explica a presença do $\mathrm{SO}_{3}$ na amostra do coating.

Tabela 2. Composição química da amostra de coating retirada de uma panela sob condições normais de processo (coating STD). \% em massa

\begin{tabular}{ccccccc}
\hline Amostra & $\mathrm{CaO}$ & $\mathrm{SiO}_{2}$ & $\mathrm{MgO}$ & $\mathrm{SO}_{3}$ & $\mathrm{Al}_{2} \mathrm{O}_{3}$ & $\mathrm{Fe}_{2} \mathrm{O}_{3}$ \\
\hline $\begin{array}{c}\text { Coating } \\
\text { STD }\end{array}$ & 61,59 & 32,48 & 3,41 & 1,19 & 0,58 & 0,71 \\
\hline
\end{tabular}

As previsões termodinâmicas realizadas durante a investigação da dissolução da camada protetora de $\mathrm{C}_{2} \mathrm{~S}$ em função do teor de fluorita (conforme tabela 1) estão representadas nas figuras $1,2,4$ a 6 ).

A evolução das transformações de fases da amostra $A$, sem adição de fluorita, (Figura 1), revela que na temperatura de $1600^{\circ} \mathrm{C}$ há $15 \%$ em massa de fase líquida (Slag) e $85 \%$ de fase sólida $\left(\mathrm{C}_{2} \mathrm{~S}\right)$. Segundo a literatura [4], as temperaturas solidus (formação do primeiro líquido) e liquidus (temperatura de fusão) do $\mathrm{C}_{2} \mathrm{~S}$ alfa puro são de aproximadamente 1464 e $2154^{\circ} \mathrm{C}$, no entanto, de acordo com a Figura 1 , acima de $1200^{\circ} \mathrm{C}$, inicia-se a formação de fase líquida. Esta diferença pode ser explicada em função da presença de contaminantes na amostra. A solução sólida de periclásio $(\mathrm{MgO})$ pode dissolver alguma quantidade de $\mathrm{CaO}$.

As transformações polimórficas do $\mathrm{C}_{2} \mathrm{~S}$ na simulação (Figura 1) mostram o polimorfo de menor temperatura $\mathrm{Y}$-C2S (sistema ortorrômbico), estável a temperatura ambiente, transformando-se, entre $500^{\circ} \mathrm{C}$ e $800^{\circ} \mathrm{C}$, no polimorfo a prime e acima de $1425^{\circ} \mathrm{C}$ no polimorfo $\alpha$. Segundo Mumme et al. [13] o resfriamento a $670^{\circ} \mathrm{C}$ produz o polimorfo $\beta$ (monoclínico-metaestável), o qual não se forma durante o aquecimento a partir do polimorfo $\gamma$. Por sua vez $\beta$, com resfriamento a temperaturas inferiores a $500^{\circ} \mathrm{C}$, converte-se de forma reconstrutiva e irreversível para $\vee$ (Figura 3).

\footnotetext{
* Contribuição técnica ao 450 Seminário de Aciaria - Internacional, 25 a 28 de maio de 2014 Porto Alegre, RS, Brasil.
} 
A diferença no comportamento polimórfico dos dados da literatura e dos dados do programa FactSage é explicada pelo fato do programa considerar nos cálculos o equilíbrio termodinâmico, portanto, fases metaestáveis como o polimorfo $\beta$ não são consideradas pelo programa.

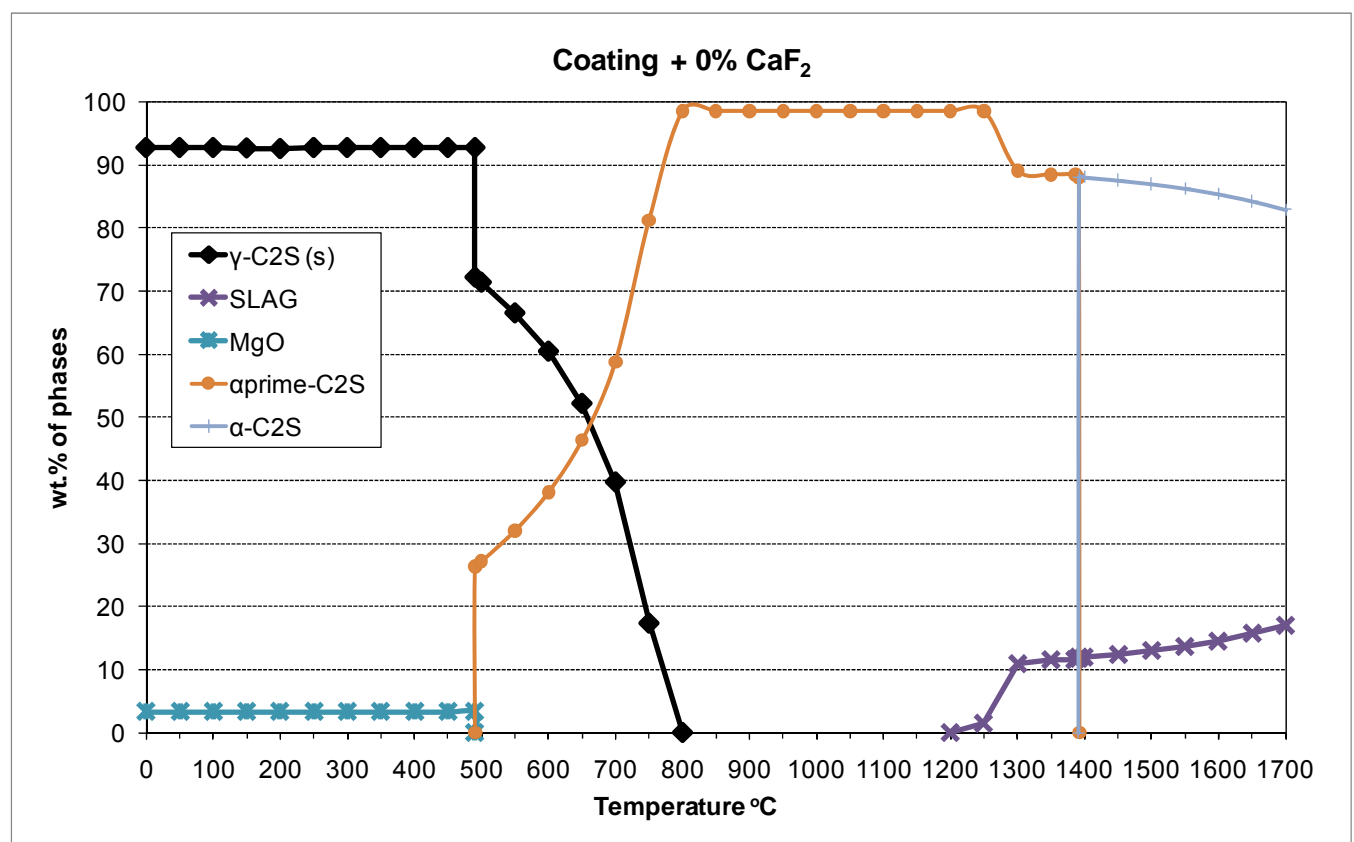

Figura 1. Evolução das fases do $\mathrm{C}_{2} \mathrm{~S}$ quando aquecido em atmosfera redutora. Dados gerados no FactSage.

A adição de $5 \%$ em massa de fluorita (amostra B) aumenta para 55\% em massa a quantidade de fase líquida (Slag) na temperatura de $1600^{\circ} \mathrm{C}$ (Fig.2). A formação de fase líquida nesta composição inicia-se a $1100^{\circ} \mathrm{C}$. $\mathrm{O}$ grande aumento no percentual de líquido é explicado pela formação da fase cuspidina $\left(\mathrm{Ca}_{4} \mathrm{Si}_{2} \mathrm{~F}_{2} \mathrm{O}_{7}\right)$, que apresenta baixo ponto de fusão $\left(\sim 1250^{\circ} \mathrm{C}\right)$ e favorece a formação da fase líquida.

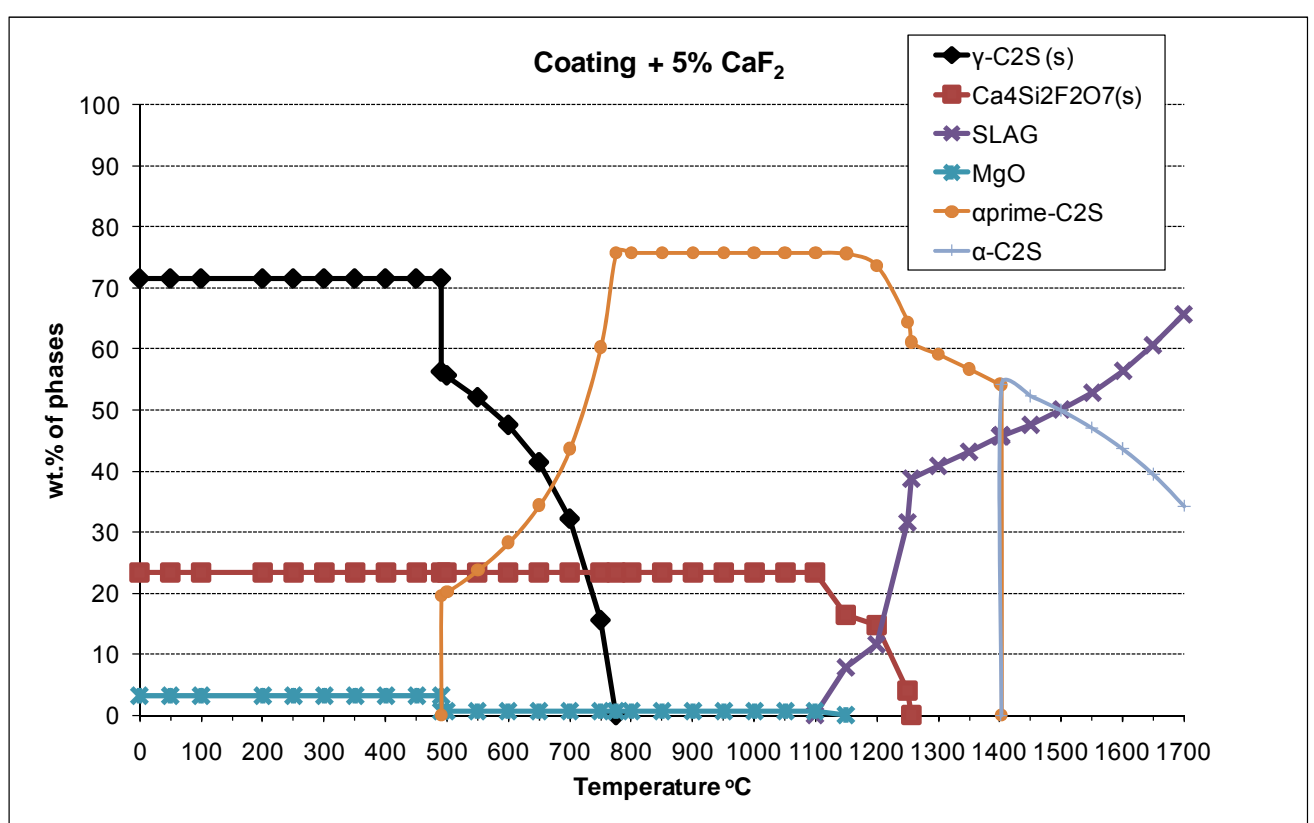

Figura 2. Processo de evolução das fases formadas na mistura $\left(\mathrm{C}_{2} \mathrm{~S}+5 \% \mathrm{CaF}_{2}\right)$ como função da temperatura. Dados gerados no FactSage.

* Contribuição técnica ao $45^{\circ}$ Seminário de Aciaria - Internacional, 25 a 28 de maio de 2014, Porto Alegre, RS, Brasil. 


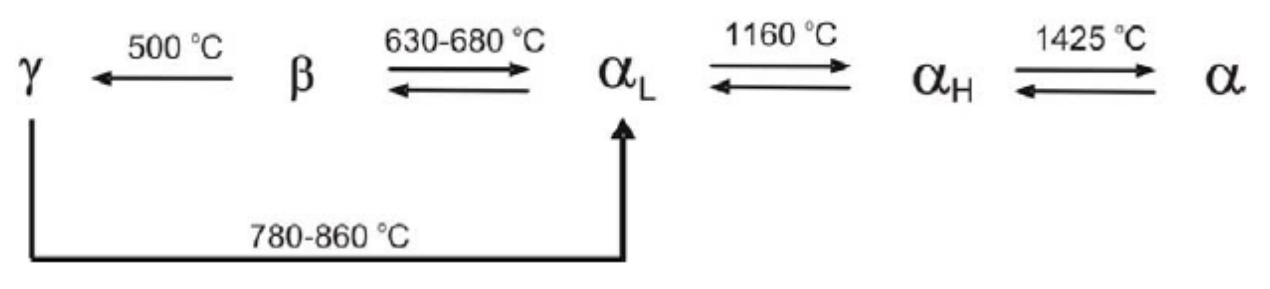

Figura 3. Temperaturas de transformações polimórficas do C2S, sob pressão atmosférica [13].

O aumento do teor de fluorita para 10\% em massa (amostra C) aumenta para $90 \%$ em massa a quantidade de fase líquida (Slag) na temperatura de $1600^{\circ} \mathrm{C}$. $\mathrm{O}$ aumento no teor de fluorita do sistema $\mathrm{C}_{2} \mathrm{~S}-\mathrm{CaF}_{2}$ favorece a formação da fase cuspidina e consequentemente a formação de fase líquida em temperaturas inferiores. Nesta composição, a formação de fase líquida tem início abaixo de $1000^{\circ} \mathrm{C}$. Destaca-se a formação da fase $\mathrm{CaF}_{2}(\mathrm{~s})$, sugerindo o início de saturação da fluorita no sistema $\mathrm{C}_{2} \mathrm{~S}-\mathrm{CaF}_{2}$.

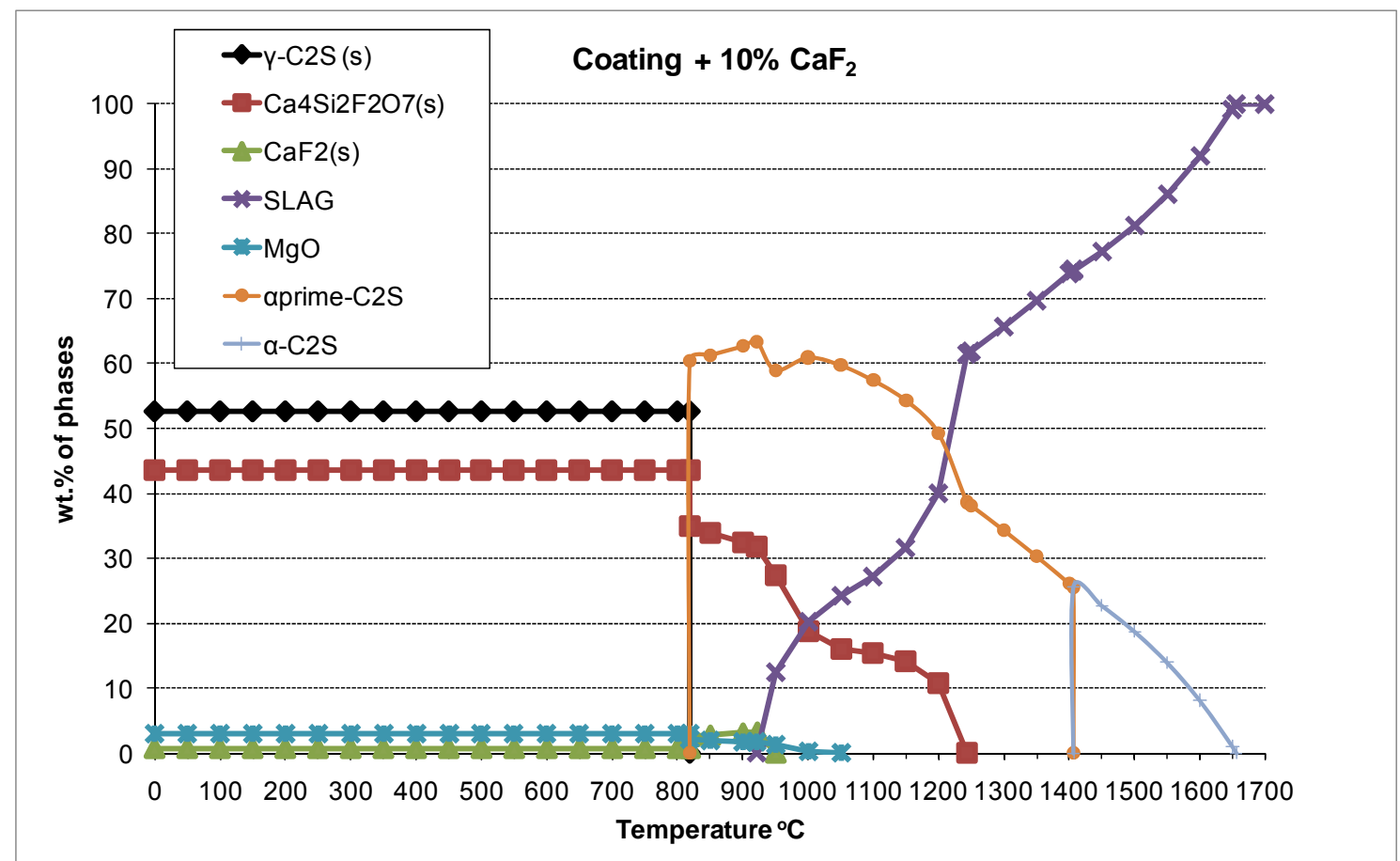

Figura 4. Processo de evolução das fases formadas na mistura $\left(C_{2} S+10 \% \mathrm{CaF}_{2}\right)$ como função da temperatura. Dados gerados no FactSage.

Aumentando-se o teor de fluorita para $20 \%$ em massa no sistema $\mathrm{C}_{2} \mathrm{~S}-\mathrm{CaF}_{2}$ (amostra D) $100 \%$ em massa de fase líquida (Slag) é obtida acima de $1200^{\circ} \mathrm{C}$. Nesta concentração o teor de fluorita está supersaturado e além da presença da fase cuspidina é possível identificar cerca de $10 \%$ em massa de fluorita que não reagiu. Portanto, de acordo com as Figuras 4 e 5 a saturação de fluorita no sistema $\mathrm{C}_{2} \mathrm{~S}-\mathrm{CaF}_{2}$ ocorre próximo a $10 \%$ em massa de fluorita e acima desta concentração não há aumento na proporção da fase cuspidina e sim da fluorita não reagida.

\footnotetext{
* Contribuição técnica ao 450 Seminário de Aciaria - Internacional, 25 a 28 de maio de 2014, Porto Alegre, RS, Brasil.
} 


\section{ACIARIA

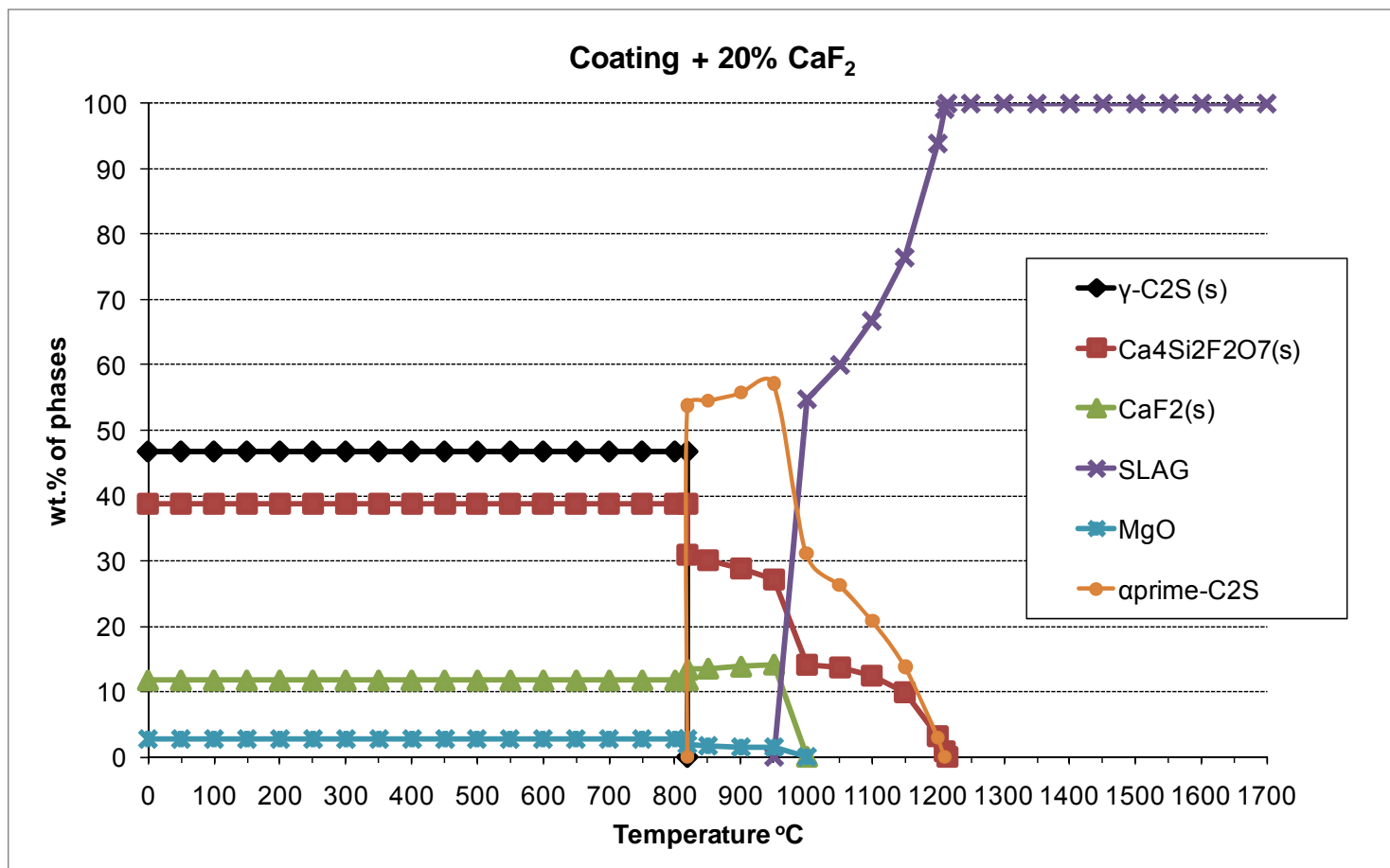

Figura 5. Processo de evolução das fases formadas na mistura $\left(\mathrm{C}_{2} \mathrm{~S}+20 \% \mathrm{CaF}_{2}\right)$ como função da temperatura. Dados gerados no FactSage.

Apesar da grande importância industrial o uso da fluorita exige muitos cuidados. A Tabela 3 apresenta resumidamente o grande potencial da fluorita como agente fluxante no sistema $\mathrm{C}_{2} \mathrm{~S}-\mathrm{CaF}_{2}$. Os resultados indicam que pequenas concentrações de $\mathrm{CaF}_{2}$ são capazes aumentar consideravelmente a proporção de líquido no sistema: $10 \%$ de $\mathrm{CaF}_{2}$ no sistema $\mathrm{C}_{2} \mathrm{~S}-\mathrm{CaF}_{2}$ é suficiente para dissolver $90 \%$ do sistema na temperatura de $1600^{\circ} \mathrm{C}$.

Tabela 3. Temperaturas liquidus e solidus do sistema $\mathrm{C}_{2} \mathrm{~S}-\mathrm{CaF}_{2}$ em função do aumento do teor de $\mathrm{CaF}_{2}$. Percentagem em massa

\begin{tabular}{ccccc}
\hline Amostra & Composição & $\begin{array}{c}\text { Temperatura } \\
\text { líquidus }\left({ }^{\circ} \mathrm{C}\right)\end{array}$ & $\begin{array}{c}\text { Temperatura } \\
\text { sólidus }\left({ }^{\circ} \mathrm{C}\right)\end{array}$ & $\begin{array}{c}\% \text { Líquido } \\
\left(1600^{\circ} \mathrm{C}\right)\end{array}$ \\
\hline $\mathrm{A}$ & $100 \%-\mathrm{C}_{2} \mathrm{~S}$ & $>>1700$ & 1200 & 15 \\
$\mathrm{~B}$ & $95 \% \mathrm{C}_{2} \mathrm{~S}-5 \% \mathrm{CaF}_{2}$ & $>>1700$ & 1100 & 55 \\
$\mathrm{C}$ & $90 \% \mathrm{C}_{2} \mathrm{~S}-10 \% \mathrm{CaF}_{2}$ & 1650 & 950 & 90 \\
$\mathrm{D}$ & $80 \% \mathrm{C}_{2} \mathrm{~S}-20 \% \mathrm{CaF}_{2}$ & 1200 & 950 & 100 \\
\hline
\end{tabular}

Os dados apresentados na Tabela 3 estão representados graficamente na Figura 6. Assim, é possível uma análise global e comparativa da evolução de formação de fase líquida em função do aumento da temperatura e teor de fluorita no sistema $\left(\mathrm{C}_{2} \mathrm{~S}+\mathrm{CaF}_{2}\right)$. Embora já comentado anteriormente (Tabela 3), fica claro na Figura 6 o potencial de dissolução da fluorita.

\footnotetext{
* Contribuição técnica ao 450 Seminário de Aciaria - Internacional, 25 a 28 de maio de 2014, Porto Alegre, RS, Brasil.
} 

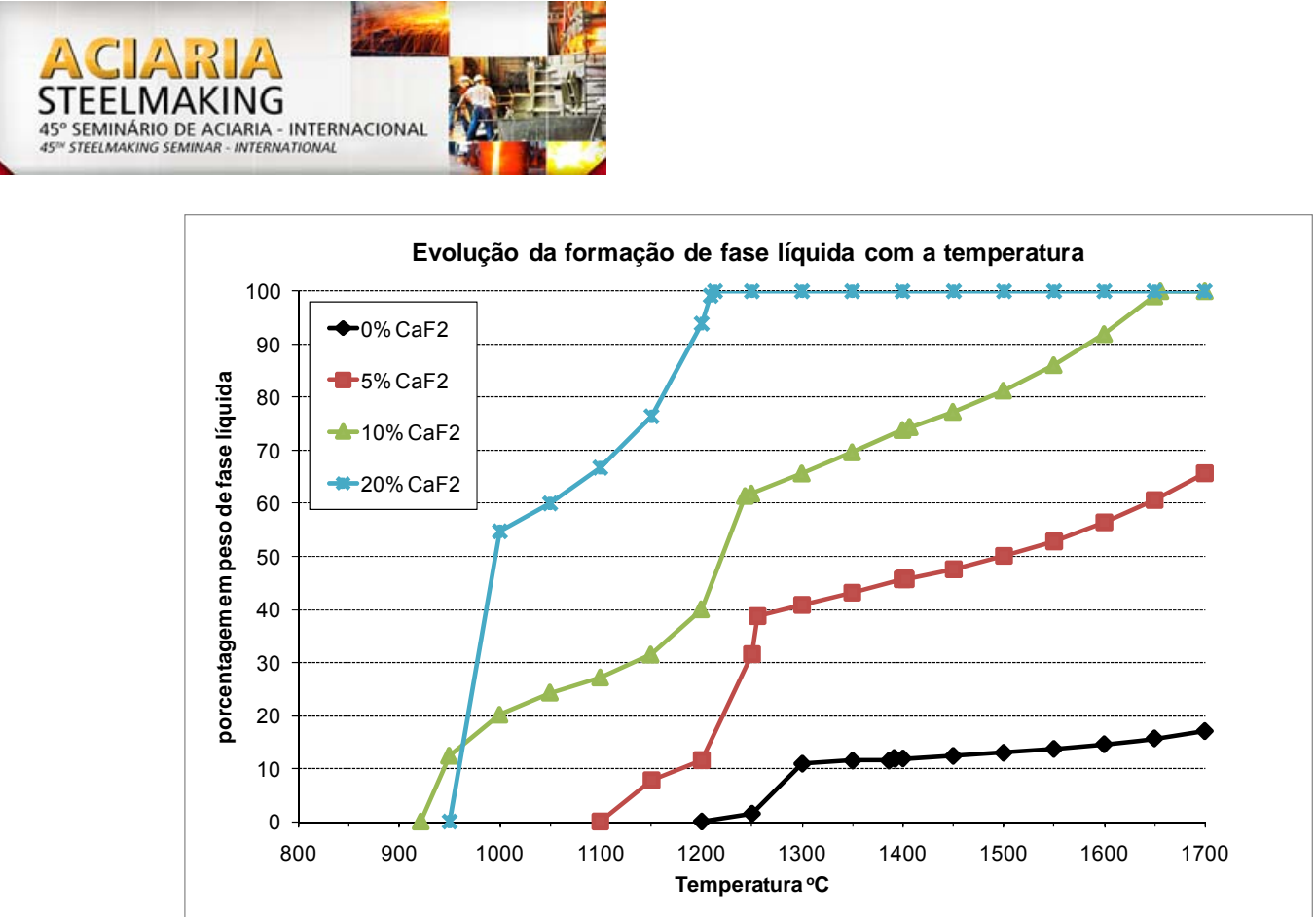

Figura 6. Evolução da formação de fase líquida em função do aumento da temperatura e teor de fluorita no sistema $\left(\mathrm{C}_{2} \mathrm{~S}+\mathrm{CaF}_{2}\right)$. Dados gerados no FactSage.

Paralelamente ao estudo de simulação termodinâmica foram realizados testes experimentais (com as mesmas composições) para avaliar a evolução das fases formadas em função do teor de fundente e validar os resultados obtidos no programa FactSage.

Os resultados da evolução de formação de fases do teste experimental (Figura 7) indicam uma grande aproximação com as fases obtidas através da simulação termodinâmica. De acordo com a análise de DRX, foram identificadas as seguintes fases no teste experimental: $\mathrm{Y}-\mathrm{C}_{2} \mathrm{~S}$ (cristaliza-se no sistema ortorrômbico, com estrutura análoga à do mineral Olivina), Cuspidina $\left(\mathrm{Ca}_{4} \mathrm{Si}_{2} \mathrm{~F}_{2} \mathrm{O}_{7}\right)$, Periclasio $(\mathrm{MgO})$ e Fluorita $\left(\mathrm{CaF}_{2}\right)$. As fases $\mathrm{Y}-\mathrm{C}_{2} \mathrm{~S}$, Periclásio e Cuspidina estão presentes em todas as composições. O aumento da intensidade do pico de Cuspidina (29 graus) indica um aumento na proporção desta fase, em função do aumento de Fluorita. Além disso, observa-se o aumento da proporção da fase fluorita (28 graus) com o aumento da própria fluorita no sistema, indicando a presença de fluorita não reagida.

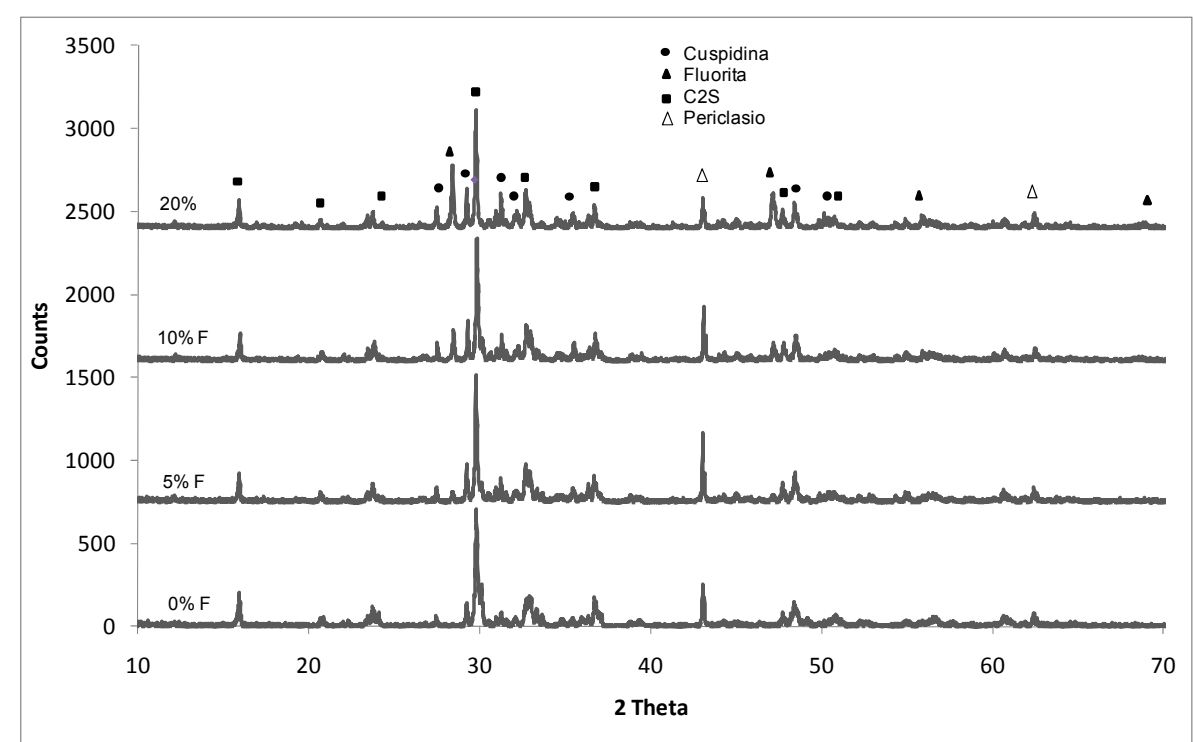

Figura 7. Processo de evolução das fases formadas no sistema $\left(\mathrm{C}_{2} \mathrm{~S}-\mathrm{CaF}_{2}\right)$ em função do aumento do teor de fluorita. Amostras submetidas a aquecimento até a temperatura de $1600^{\circ} \mathrm{C}$ em atmosfera inerte com posterior resfriamento até a temperatura ambiente.

* Contribuição técnica ao $45^{\circ}$ Seminário de Aciaria - Internacional, 25 a 28 de maio de 2014, Porto Alegre, RS, Brasil. 


\section{CONCLUSÕES}

Pequenas concentrações de $\mathrm{CaF}_{2}$ são capazes aumentar consideravelmente a proporção de líquido quando em contato com a camada protetora aderida aos refratário doloma-C (Silicato Dicálcico $-\mathrm{C}_{2} \mathrm{~S}$ ).

Teores de $5 \%$ e $10 \%$ em massa de $\mathrm{CaF}_{2}$ no sistema $\mathrm{C}_{2} \mathrm{~S}-\mathrm{CaF}_{2}$ são capazes de formar $55 \%$ e $90 \%$ de fase líquida respectivamente na temperatura de $1600^{\circ} \mathrm{C}$. Além disso, a presença de Fluorita diminui consideravelmente a temperatura inicial de fusão durante o aquecimento (linha solidus).

$\mathrm{O}$ aumento da fase líquida em função do teor de $\mathrm{CaF}_{2}$ é justificável pela presença da fase Cuspidina e Fluorita, analisadas por difração de raios- $X$, as quais apresentam baixas temperaturas de fusão, favorecendo assim maior proporção de líquido na temperatura de $1600^{\circ} \mathrm{C}$.

Os resultados experimentais e bibliografias existentes mostraram coerência com os dados das simulações. As transformações polimórficas do $\mathrm{C}_{2} \mathrm{~S}$ propostas pela simulação são coerentes com os dados encontrados na literatura. Além disso, as fases encontradas na análise por difração de raios- $X$ são as mesmas obtidas na simulação.

\section{REFERÊNCIAS}

1 Nakahara T. Innovation in a Borderless World Economy. Research \& Technology Management. 1997. p.7-9.

2 Schacht, CA. Refractories Handbook. Marcel Decker; 2004.

3 Lee WE, Zhang S. Melt corrosion of oxide-carbon refractories. International Materials Reviews.v.44, n.3. Sheffield, UK: Communications Ltd and ASM International,1999. p. 309-320.

4 Eriksson G, Wu P, Blander M, Pelton AD. Critical evaluation and optimization of the thermodynamic properties and phase diagrams of the $\mathrm{MnO}-\mathrm{SiO} 2$ and $\mathrm{CaO}-\mathrm{SiO} 2$ systems. Can. Metall. Q. 1994;33:13-21.

5 Lee WE, Zhang S. Direct and indirect slag corrosion of oxide and oxide-c refractories. In: VII International Conference on Molten Slags Fluxes and Salts; 2004. The South African Institute of Mining and Metallurgy; 2004.

6 Jung I-H, Van Ende MA, Kim DG. Thermodynamic database development for CaF containing oxide systems and applications to the steelmaking process. Montreal: Dept. Mining and Materials Engineering, McGill University; 2012.

7 Herbert W, Heinz-Gunter S, Herbert N. Refractory wear and steel desulfurization during vacuum degassing with controlled fluorspar additions. Metallurgical Plant and Technology International. 1991;5:50-59.

8 Factsage [página da internet]. Disponível em: http://www.factsage.com.

9 Bale CW, Chartrand P, Degterov SA, Eriksson G, Hack K, Ben Mahfoud R, Melançon J, Peltron $A D$, Petersen $S$. FactSage thermochemical software and databases. Calphad. 2002;26(2):189-228.

10 Bale CW, Bélisle E, Chartrand P, Degterov SA, Eriksson G, Hack K, Jung I-H.; Kang YB, Melançon J, Pelton AD, Robelin C, Petersen S. Calphad. 2009;33:295-311.

11 FactSage Database Documentation. Part integrant of FactSage software version 6.3 and revised; 2012.

12 Gomes SD. Incorporação de enxofre no silicato dicálcico do clínquer de cimento Portland: influência na estabilidade de polimorfos e na reatividade hidráulica [dissertação de mestrado].São Paulo: Universidade de São Paulo; abril de 2007.

13 Mumme WG, Hill RJ, Bushnell-Wye G, Segnit ER. Rietveld crystal structure requirements, crystal chemistry and calculated powder diffraction data for the polymorphs of dicalcium silicate and related phases. N. Jb. Miner. Abh. 1995;169:35-68.

* Contribuição técnica ao 45 Seminário de Aciaria - Internacional, 25 a 28 de maio de 2014, Porto Alegre, RS, Brasil. 